\title{
Colciencias e Inconciencias con los Científicos Colombianos: de la Edad de Piedra al Factor de Impacto
}

\author{
Fidias Leon-Sarmiento $^{1}$, Jaime Bayona-Prieto ${ }^{2}$, Edgardo Bayona ${ }^{3}$ y Martha \\ León ${ }^{4}$ \\ ${ }^{\mathbf{1}}$ Médico Cirujano, Especialista en Neurología Clínica, Epidemiología y Docencia Universi- \\ taria, Ph. D. Neurofisología Clínica. Grupo Neuro.net-Neurología Clínica y Funcional, Neu- \\ robiología Humana \& Neurociencias Aplicadas; Universidad de Pamplona, Colombia. E- \\ mail: feleoness@yahoo.com \\ ${ }^{2}$ Fisioterapeuta, Especialista en Neurorehabilitación., Ph. D. (Candidato) en Neurociencias. \\ Grupo Neuro.net-Neurología Clínica y Funcional, Neurobiología Humana \& Neurociencias \\ Aplicadas; Universidad de Pamplona, Colombia. E-mail: jaimebayona@unipamplona.edu.co \\ ${ }^{3}$ Universidad Antonio Nariño, Bogota. Grupo Neuro.net-Neurología Clínica y Funcional, \\ Neurobiología Humana \& Neurociencias Aplicadas, Colombia. \\ E-mail: edgardobayona@yahoo.com \\ ${ }^{4}$ Economista, M. Sc., Ph. D. (Candidata) en Gerencia. Grupo Neuro.net-Neurología Clínica y \\ Funcional, Neurobiología Humana \& Neurociencias Aplicadas, Colombia. \\ E-mail: maedles22@yahoo.com
}

Recibido 22 Febrero 2005/Enviado para Modificación 15 Abril 2005/Aceptado 162005

\section{RESUMEN}

La evolución de la escritura, en el mundo, ha sido dramática; sin embargo, la evaluación de producción científica en Colombia no, incluyendo el mal uso de los decretos gubernamentales 1444 de 1993 y 1279 del 2002. Este último autorizó a Colciencias, ente creado para apoyar la investigación en Colombia, a reglamentar la evaluación de la producción intelectual, quien determinó evaluarla con el nada científico "factor de impacto", y descalificó a MEDLINE/PubMed y PsyINFO, sin justificación alguna, acercándose con esto al prevaricato. Esto afecta las investigaciones colombianas y el salario de los docentes-investigadores dejando, además, en gran desventaja las publicaciones científicas colombianas. Recomendamos que manuscritos registrados en bases de datos calificadas, incluyendo PubMed/MEDLINE, se califiquen de acuerdo a la ley, evitando así un mayor perjuicio a la producción científica escrita colombiana.

Palabras Clave: Factor, impacto, investigación, Colombia (fuente: DeCS, BIREME) 


\section{ABSTRACT \\ Colciencias and disdain for Colombian scientists: From the Stone Age to the Impact Factor}

Writing has dramatically evolved in the world; however, qualification of scientific production in Colombia has not, including the improper use of decree $1444 / 93$ and $1279 / 02$. The last of these decrees authorized Colciencias, the Colombian government institute created to support scientific research in Colombia, to establish rules for its implementation. Colciencias decided to evaluate scientific papers produced in Colombia based on the non-scientific method of the "impact factor", and considered that citations in MEDLINE/PubMed and PsyINFO were second line publications thus violating Colombian law. This affects not only the progress of scientific research in Colombia but also researchers' income and puts Colombia's scientific journals and publications at great disadvantage. Scientific papers indexed in qualified databases such as MEDLINE/PubMed must be judged according to law in order to prevent further injuries to the developing Colombian scientific production.

Key Words: Impact factor, research, Colombia (source: MeSH, NLM).

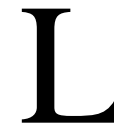

a dramática evolución de la escritura desde la hecha, hasta hace unos años en escuelas colombianas, en piedra y apoyada por el ministerio de educación (Figura 1), hasta la actual transmisión electrónica de datos ha afectado las publicaciones científicas en Colombia. Allí se consideró, hasta hace poco, que los especialistas poseían el máximo nivel académico, principalmente en medicina. Ellos eran mas que todo, trasmisores de datos, con escasa producción escrita que siguiera el poco útil, aunque unificado, método científico (1), encontrándose facultades de medicina que generaron solo un manuscrito en MEDLINE, en 20 años de labores (2).

A finales de 1980 aumentaron los maestros y Ph Ds en todas las áreas, así como las publicaciones científicas relacionadas con Colombia (3). Por ello y para motivar, aun más, la producción científica, el gobierno colombiano expidió, a finales del siglo pasado, el decreto 1444 del 1993, con sus límites y beneficios (4). Luego, para "modernizar" e "incentivar mucho mas" dicha producción y su evaluación expidió el decreto 1279 del 2002 (5). 


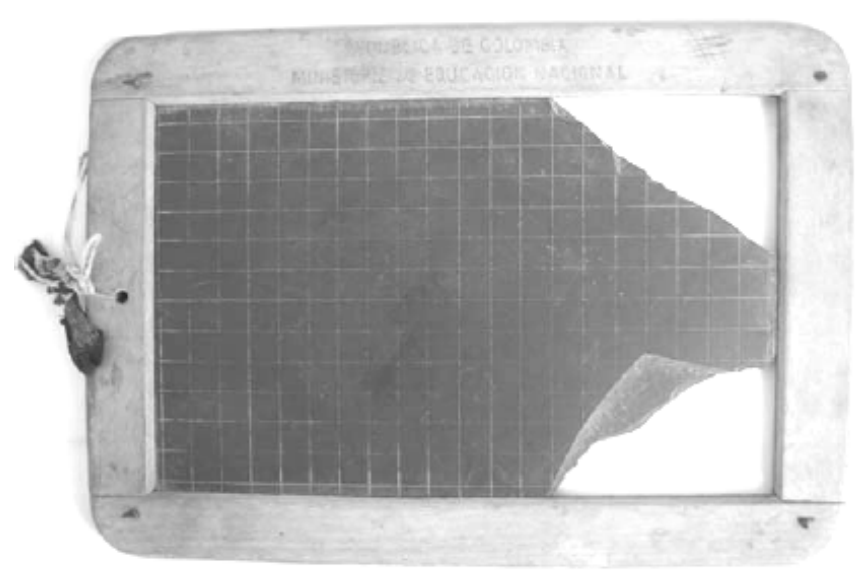

Figura 1. Pizarra hecha en piedra, utilizada por estudiantes de primaria a principios del siglo $X X$, en Colombia. Esta cara de la pizarra, con cuadrícula inserta, era para estudiar "matemáticas", el reverso sin cuadrícula se utilizaba para estudiar otras asignaturas como "lenguaje y sociales". En la parte superior está escrito: "REPÚBLICA DE COLOMBIA, MINISTERIO DE EDUCACION NACIONAL ". Las esquinas del lado derecho se encuentran quebradas.

Discutiremos a continuación el pasado y presente de algunos de estos tópicos, incluyendo algunos ejemplos de universidades colombianas, esperando que esto sirva de fundamento para que, en un futuro cercano, la ciencia colombiana realmente impacte local, regional e internacionalmente; enfatizando que dicho impacto no deberá basarse en manipulaciones y oscilaciones particulares, sino en principios más realistas y ajustados a un verdadero criterio científico y académico que el que existe a la fecha.

\section{EL PASADO}

El desconocimiento de lo que era investigar y publicar científicamente, generó múltiples vacíos en el decreto 1444 de 1993 (4), "llenados" por los comités de puntaje de universidades colombianas con diversas interpretaciones, debido a la falta de experiencia de algunos de sus integrantes para calificar artículos científicos. Unos no sabían que era lo que calificaban, como aconteció en la UIS de Bucaramanga; mientras que otros daban una interpretación ambigua, como ocurrió en UniPamplona.

Este decreto afianzó, además, el concepto endofenotípico de " lo grande" del trabajo o " la longitud" de la bibliografía o "el grosor" de la tesis a presentar y por ende su importancia. Cartas al editor, comunicaciones breves o 
editoriales, publicados e indizados en excelentes bases de datos internacionales se descalificaron a priori, opuesto a lo estatuido (4).

Cabe recordar que cartas al editor o manuscritos "cortos", muchas veces, han cambiado paradigmas científicos (6), como sucedió con Watson y Crick en 1953, quienes sentaron las bases de las investigaciones biológicas moleculares modernas (7), o Patarroyo y su grupo y sus investigaciones en malaria $(8,9)$. Sin embargo, comités de puntaje de algunas universidades colombianas sostienen que estas publicaciones carecen de importancia, como lo afirma la Dra. L.A. Patiño (Secretaria General Universidad Industrial de Santander, comunicación personal, Agosto 25, 1998), lo cual sirve de base para descalificar publicaciones como las hechas en Toxicology letters, Immunology letters o Neuroscience Letters entre otras, quienes tienen un elevado nivel científico y alto grado de dificultad para lograr la aceptación de manuscritos.

De todos es conocido que entre mas pequeña es la escritura en piedra, mayor es la dificultad para maniobrarla y, por ende, más valor tendrá la obra esculpida allí. Por esto Victor Frankl, padre de la logoterapia clínica, afirmaba que "No es la duración de una vida humana en el tiempo la que determina la plenitud de su sentido", y que "No deberíamos juzgar el valor de una biografía por su extensión (o tamaño), por el número de páginas del libro, sino por la riqueza se contenido” (paréntesis de los autores) (10).

Además de las anteriores malinterpretaciones, algunos claustros colombianos como la UIS de Bucaramanga, re-evaluaban manuscritos ya evaluados por pares y publicados en revistas de alto nivel científico. Dichas reevaluaciones internas se cuestionaron por la calidad que los "nuevos pares" asignados tenían de los temas a re-evaluar, además de la aplicación de una particular operación matemática, llevando todo esto, en ocasiones, a descalificar por completo algunos trabajos. Lo anterior violaba el "debido proceso" a los profesores-investigadores, porque nadie puede ser juzgado dos veces por el mismo hecho o falta; llamándose falta al publicar o investigar, visto así por algunas autoridades de entonces. El artículo 8 del código penal colombiano claramente prohíbe la doble incriminación y dice: "A nadie se le podrá imputar mas de una vez la misma conducta...” independiente de “... la denominación jurídica que se le haya dado...”, o “non bis in idem”. 


\section{EL PRESENTE}

Pretendiendo subsanar los vacíos del decreto 1444 de 1993, el gobierno Colombiano re-reglamentó la evaluación de la producción científica universitaria y expidió el decreto 1279 del 2002 (5). Este le dio superpoderes a Colciencias, ente creado para apoyar la investigación en Colombia, para que reglamentara la evaluación de la producción científica colombiana, nacional e internacional, haciendo esto último después de muchos meses de expedido el decreto (11), perjudicando el salario de los docentes y llevando a algunas universidades a aplicar, ilegalmente, el decreto 1444 de 1993. Los exabruptos cometidos con la indización de las publicaciones colombianas se comentaron previamente (12).

Desafortunadamente, las nuevas directrices emanadas de Colciencias no se correlacionan con el decreto 1279 del 2002 (5), porque en su capítulo V, artículo 24, numeral I, letra (a) dice: "Según los criterios de Colciencias, se clasifican, indexan u homologan las revistas especializadas indexadas internacionalmente, en los tipos A1 y A2. Para las demás revistas que cumplan los criterios de Colciencias, esta institución las clasifica, indexa u homologa en los tipos B y C”. Sin embargo, Colciencias clasificó en la categoría mas alta llamada A1 -a las que la ley colombiana asigna 15 puntos- las revistas del Journal Citation Report (JCR), que estén en el $25 \%$ superior del conjunto de revistas de la especialidad; las que se ubiquen por debajo del $75 \%$ de dicho conjunto de revistas estarán en la categoría A2. Con gran inconciencia basada en estas evidencias, Colciencias ubicó en la categoría B - a las que la ley colombiana asigna 8 puntos - las bases de datos MEDLINE/PubMed y PsyINFO, desconociendo su importancia científica y el esfuerzo requerido para publicar en ellas, violando con esta clasificación "a dedo” la ley colombiana y afectando además los ya, de por sí, exiguos ingresos de los profesores universitarios estatales colombianos. Excepto Scielo, otras numerosas bases de datos con gran calidad científica, ni siquiera los mencionó Colciencias (11).

Mas significativo fue el que Colciencias clasificara las revistas internacionales basada en el mal llamado factor de impacto (FI), utilizado por el JCR y su aliado el Current Contents y que está siendo reevaluado y tomando visos de corrupto, a nivel mundial (13-19), dejando de lado factores mas importantes como "equidad” y "calidad", entre otros. Esto confirma la idiosincrasia regional de usar desechos intelectuales de otros países, que frenan, aún mas, el desarrollo científico de la nación. Dicho FI es, además, sectáreo, de estrecha visión y refleja una pintoresca variedad de filias, fobias, ignorancia, mitos y rituales (20); se favorece por y acepta mas las publicaciones en 
inglés (20), lo que es discriminatorio y lesiona el principio de igualdad en Colombia al privilegiar la producción científica anglosajona y rezaga la hecha en español, por esta competencia desigual, aunque esta última se evalue con toda la rigurosidad científica (21).

Por tal motivo, Benitez-Bibriesca, editor de Archives of Medical Research afirma que "El FI es un indicador útil para bibliotecarios, distribución de revistas y aún mercadeo pero muy diferentes deben ser los criterios que se deben aplicar a la evaluación de la calidad de las publicaciones científicas" (22). Así, el uso y aplicación patológica del FI, manipulado por el JCR y sus aliados, quienes manejan grandes sumas de dinero, acabará con publicaciones regionales, fruto de la globalización de las publicaciones científicas (21).

El mismo JCR dice que "The impact factor is a very useful tool for evaluation of journals, but it must be used discreetly"; debiendo evitarse falsos significados dado que "discreetly" es mas que "discreto" en español; y que él no depende sólo del FI para evaluar la utilidad de una revista "....and neither should anyone else” (23), lo que debe incluir a Colciencias y similares.

Lo anterior se debe a que diversos factores modifican la citaciones científicas. Por ejemplo, las revisiones "...se citan con mas frecuencia que los artículos típicos de investigación..." (23); los "artículos de investigación originales con mas de 100 referencias," el JCR los toma como "revisión" (23) y "naturally, review journals have some of the highest impact factors" (23). Tal es el caso de la revista Annual Review of Public Health quien tuvo en el 2000 un FI de 4,524, mayor a los FI de las revistas que publican investigaciones -mal llamadas- originales como American Journal of Epidemiology y American Journal of Public Health (20). Curiosamente, para realizar revisiones científicas, un investigador tiene que publicar primero numerosos artículos en revistas de bajo impacto (24). El JCR afirma, también, que las "revistas dedicadas a publicar métodos de investigación usualmente no tienen alto impacto" (25). O que, áreas como las "artes y humanidades” toman un largo tiempo "mientras algún artículo logra tener un número significativo de citaciones" (25). Las citaciones se afectan, también, por el "efecto placebo" producido por alguien con poder en el área científica. Tales inexactitudes producen fluctuaciones de los FI, generando situaciones como la acontecida con la revista Advances in Nuclear Physics que pasó del 9 al 261 puesto entre 1990 y 1995 o Lancet que pasó de un FI de 17,490 a 10,232 de 1995 al 2000; particularmente, las cartas al editor aumentan el FI (26).

Estos detalles, al parecer, no fueron analizados por Colciencias originándose confusión con la norma basada en el FI al punto que, revistas indizadas 
en MEDLINE/PubMed y PsyINFO se califican muy por debajo de otras con circulación local y menor trascendencia. De particular interés es que MEDLINE/PubMed se utiliza sistemáticamente para efectuar los mejores y mas actualizados estudios científicos meta-analíticos, fundamento de decisiones clínicas, diagnósticas y terapéuticas mundiales (27-31), incluyendo el DSM IV (32). El uso del JCR y su aliado "Current Contents" en investigaciones similares es prácticamente nula, algo muy curioso en estos tiempos de ciencia basada en evidencias. Por eso es que el mismo Eugene Garfield, creador del FI, dice que éste no es un buen indicador cienciométrico(20).

\section{EL FUTURO}

Nuevas formas de evaluación de la producción científica como las empleadas en países que han rechazado la manipulación del FI orquestado por el JCR, como Alemania, Holanda e Inglaterra, quienes poseen métodos de evaluación mas autóctonos y contextualizados, deben explorarse para Colombia y pronto. Por ello, con lo estatuido a la fecha, proponemos lo siguiente:

Colocar en la categoría A1 las revistas que aparezcan en índices científicos mundiales, no sólo biomédicos, incluyendo allí a MEDLINE/PubMed y PsyInfo, resaltando que, por ejemplo, filósofos, artistas y textiles, tienen sus propias bases de datos igualmente serias. En la Categoría A2, se incluirían revistas indizadas de forma regional como Scielo, dirigido a captar revistas hispanoamericanas, para nombrar solo uno de ellos. También aquí podrían caber LILACS, BIREME y similares. Esto facilitaría la evaluación de científicos foráneos que arriben al país con, probablemente, publicaciones en índices científicos internacionales serios pero con circulación restringida en sus continentes. Las demás revistas que cumplan los criterios de ley, se ubicarían en las categorías $\mathrm{B}$ y $\mathrm{C}$, incluyéndose aquí revistas publicadas en otros países, pero que no estén indizadas en base de datos alguna, dejando en la categoría B las publicadas en Español y en C las publicadas en otro idioma, para estimular el uso de nuestra lengua en el campo científico.

Finalmente, solo en la medida en que los docentes universitarios colombianos asuman un papel mas crítico y activo, fruto de una fundamentación teórica apropiada y digna del trabajo que realizan, se logrará corregir los desafueros que pretenden imponer legos disfrazados de seudocientificos que, a la fecha, insisten en normatizar la evaluación científica colombiana. El país y los futuros investigadores lo agradecerán • 
Agradecimientos. Los autores agradecen a Ana Victoria Sarmiento, por permitirnos conocer su "cuaderno" de piedra donde realizó sus primeras letras y operaciones matemáticas, utilizado a principios del siglo XX, siendo esto un verdadero "factor de impacto“ en nuestra vida de científicos, docentes e investigadores. A Cesar E. Pabon por comentarios hechos a una versión preliminar del manuscrito.

\section{REFERENCIAS}

1. Di Trocchio F. Las Mentiras de la Ciencia. ¿Porque y como engañan los cientificos?. Madrid: Alianza; 1993.

2. Ramirez G, Pradilla G. Use of enzyme-linked immunosorbent assay in the diagnosis of cysticercosis. Arch Neurol 1987; 44: 898.

3. Roselli D, Moreno S, Maza G, Otero A. ¿Quién es Quién en la investigacion en salud en Colombia? Avanzar Forum 2001; 2: 5-15.

4. Presidencia de la República. Decreto 1444 del 3 de Septiembre de 1992, Bogotá, Colombia.

5. Presidencia de la República. Decreto 1279 del 19 de Junio de 2002 , Bogotá, Colombia.

6. Piercy MA, Sramek JJ, Kurtz NM, Cutler NR. Placebo response in anxiety disorders. Ann Pharmacother 1996; 30: 1013-9.

7. Watson JD, Crick FHC. Molecular structure of nucleic acids. Nature 1953; 171: 737-4.

8. Patarroyo ME, Romero P, Torres ML, Clavijo P, Moreno A, Martinez A, et al. Induction of protective immunity against experimental infection with malaria using synthetic peptides. Nature 1987; 328: 629-32.

9. Patarroyo ME, Amador R, Clavijo P, Moreno A, Guzman F, Romero P, et al. A synthetic vaccine protects humans against challenge with asexual blood stages of Plasmodium falciparum malaria. Nature 1988; 332: 158-61.

10. Frankl V. Psicoanalisis y Existencialismo: De la psicoterapía a la logoterapía. Mexico: Fondo de Cultura Económica; 1997.

11. Procedimiento para homologar revistas científicas extranjeras [Internet]. Disponible en: http://huitaca.colciencias.gov.co:8080/lillium/ htmlHomologacion/ index.html. Consultada en Abril 25 de 2005.

12. Hernandez CA. Los indices bibliográficos. Biomédica 2003; 23: 1-2.

13. Brookfield J. The system rewards a dishonest approach. Nature 2003; 423: 480.

14. Berghmans T, Meert AP, Mascaux C, Paesmans M, Lafitte JJ, Sculier JP. Citation indexes do not reflect methodological quality in lung cancer randomised trials. Ann Oncol 2003; 14: 715-21.

15. Hemmingsson A, Edgren J, Mygind T, Skjennald A. Use of impact factors. Lancet 2002; 359: 173.

16. Editorial. Error in citation statistics. Nature 2002; 415: 101.

17. Gallagher EJ, Barnaby DP. Evidence of methodologic bias in the derivation of the Science Citation Index impact factor. Ann Emerg Med 1998; 31: 83-6. 
18. Hecht F, Hecht BK, Sandberg AA. The journal "impact factor": a misnamed, misleading, misused measure. Cancer Genet Cytogenet 1998; 104:77-81.

19. Neuberger J, Counsell C. Impact factors: uses and abuses. Eur J Gastroenterol Hepatol 2002;14:209-11.

20. Porta M, Copete JL, Fernandez E, Alguacil J, Murillo J. Mixing journal, article, and author citations, and other pitfalls in the bibliographic impact factor. Forum 2003; 19: 1847-62.

21. Coelho PMZ, Antunes CMF, Costa HMA, Kroon EG, Sousa Lima MC, Linardi PM. The use and misuse of the "impact factor" as a parameter for evaluation of scientific publication quality: a proposal to rationalize its application. Braz J Med Biol Res 2003; 36: 1605-1612.

22. Benitez-Bibriesca L. The impact factor of medical journals: Its use and misuse. Arch Med Res 1999; 30: 161-2.

23. The impact factor [Internet]. Disponible en: http://www. Isinet.com/essays/ journalcitationreports/7.html/. Consultado Abril 25 del 2005

24. Lowy C. Impact factor limits funding. Lancet 1997; 350: 1035.

25. The ISI ${ }^{\circledR}$ Database: The Journal Selection Process [Internet]. Disponible en: http:// www. Isinet.com/essays/selectionof materialforcoverage/199701. html/. Consultado Mayo 13 del 2005

26. Brahler E, Beutel M, Decker O. Deep impact - evaluation in the sciences. Soz Praventivmed 2004; 49: 10-4.

27. Geurian KL. The cholesterol controversy. Ann Pharmacother 1996; 30:495-500.

28. Christensen AH, Gjorup T. The Helicobacter pylori theory and duodenal ulcer disease. A case study of the research process. Dan Med Bull 1995; 42: 3747.

29. Schein JR. Cigarette smoking and clinically significant drug interactions. Ann Pharmacother 1995; 29: 1139-48.

30. Leon-Sarmiento FE, Prada DG, Bayona-Prieto J, Valderrama V, Garcia I, Leon$\mathrm{S}$ ME, et al. Neurotripanosomiasis: conceptos basicos de aspectos clinicos. Biomédica 2003; 23: 462-75.

31. Editors. Addressing the limitations of structured abstracts. Ann Int Med 2004; 140: $480-1$.

32. First MB, Frances A, Pincus HA. DSM-IV-TR. Handbook of Differential Diagnosis. Washington DC: American Psychiatric Press, 2002. 